\title{
Factors Affecting Seizure Outcomes After Surgery for Cavernoma Related Epilepsy
}

\author{
Fatma OZLEN ${ }^{1}$, Cihan ISLER ${ }^{1}$, Mehmet Yigit AKGUN² ${ }^{2}$, Cigdem OZKARA ${ }^{3}$, Mert KARABACAK ${ }^{4}$, Sakir DELIL ${ }^{3}$, \\ Basak YILMAZ OZ ${ }^{3}$, Burak TAHMAZOGLU ${ }^{1}$, Mustafa UZAN ${ }^{1}$ \\ ${ }^{1}$ Istanbul University-Cerrahpasa, Cerrahpasa Medical Faculty, Department of Neurosurgery, Istanbul, Turkey \\ ${ }^{2}$ Yuksek Ihtisas Hospital, Department of Neurosurgery, Kirikkale, Turkey \\ ${ }^{3}$ Istanbul University-Cerrahpasa, Cerrahpasa Medical Faculty, Department of Neurology, Istanbul, Turkey \\ ${ }^{4}$ Istanbul University-Cerrahpasa, Cerrahpasa Medical Faculty, Medical Student, Istanbul, Turkey \\ Corresponding author: Mustafa UZAN uzan@istanbul.edu.tr
}

\section{ABSTRACT}

AIM: To present one of the largest retrospective cavernoma related epilepsy (CRE) studies which include divergent supratentorial locations operated and followed up at least 2 years. We also investigated the factors affecting the seizure outcome.

MATERIAL and METHODS: This study includes a total of 56 patients with drug-responsive $(n=40)$ and drug-resistant $(n=16)$ CRE who underwent resective surgery. Age at seizure onset, age at surgery, gender, duration of epilepsy, seizure frequencies/type before and after treatment, EEG and brain MRI findings, prescribed AEDs, preoperative and post-operative neurological status, histopathological diagnosis, post-operative seizure outcomes and surgical information were documented.

RESULTS: The average follow-up period was 69.6 months (range 24-216 months). The seizure outcome was assessed according to Engel's classification at the last follow-up. Engel class I was achieved in 53 patients (95\%); there was one patient at class II and two patients at class III. All patients in the drug-responsive group were at Engel class I after the surgery, while all patients at Engel classes II and III were in the drug-resistant patient group. This clearly shows that there were better outcomes in DRP group ( $<<0.01)$. Neither the locations of cavernomas nor the duration of epilepsy had any impact on seizure outcome $(p>0.05)$.

CONCLUSION: An earlier surgical intervention may prevent the patients from becoming drug-resistant such that their chances of being seizure free after surgery increase.

KEYWORDS: Epilepsy, Cavernous malformation, Cavernoma, Epilepsy surgery, Drug resistant epilepsy

ABBREVIATIONS: CCMs: Cerebral cavernous malformations, CRE: Cavernoma related epilepsy, EEG: Electroencephalogram, MRI: Magnetic resonance imaging, CT: Computed tomography, PET: Positron emission tomography, DVAs: Developmental venous anomalies, AEDs: Antiepileptic drugs, DRE: Drug-resistant, DRP: Drug-responsive, IOM: Intraoperative neuromonitorization

\section{INTRODUCTION}

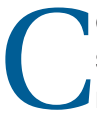
erebral cavernous malformations (CCMs) are the second most common type of vascular malformations, regarded as highly epileptogenic if they involve the cortex (6), formed by capillary dysplastic dilatations without intervening the brain parenchyma $(2,22,23)$. Epileptic seizure is the presenting symptom in almost half of the patients, $(13,17,19)$ and approximately $40 \%$ of them are resistant to drug therapy $(6,12,26)$.

Apart from seizures, cavernous malformations may cause various neurological symptoms and conditions; for example, the annual risk of hemorrhage $(11,29)$ is $0.7-1.1 \%$ in patients

\begin{tabular}{|c|c|c|c|c|c|}
\hline EN & $03-0925-8748$ & ARA & (D) : 0000-0003-0548-1838 & Basak YILMAZ OZ & (D) : 0000-0002-0955- \\
\hline & $01-8365-3338$ & Mert KARABACAK & : 0000-0002-9263-9893 & Burak TAHMAZOGLU & 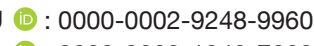 \\
\hline it AKGUN & (D) : 0000-0003-1342-7663 & Sakir DELIL & (D) : $0000-0001-5011-8929$ & Mustafa UZAN & (D) : $0000-0003-134$ \\
\hline
\end{tabular}


without a history of previous intracranial bleeding, but it may increase to up to $4.5 \%$ per year in patients who have already experienced it $(4,5,15,20)$.

Three basic surgical methods have been described for CCMs"pure lesionectomy," "lesionectomy" (excision of cavernoma and surrounding hemosiderin), and "extended lesionectomy" (i.e. anterior temporal lobectomy, tailored resection after invasive investigations, etc.). However, the optimal timing and method of surgery needs to be individualized for each patient.

In this study, we performed a retrospective analysis of patients with supratentorial CCM who underwent surgery due to cavernoma related epilepsy (CRE) and investigated the factors affecting the seizure outcomes.

\section{MATERIAL and METHODS}

This study included 56 patients who underwent resective surgery between December 2001 and January 2018 and had a histopathological diagnosis of CCM. All patients were evaluated based on non-invasive phase I investigations, including electroencephalogram (EEG) (with ictal video EEG recordings when necessary) and neuroimaging examinations such as magnetic resonance imaging (MRI), computed tomography (CT), and positron emission tomography (PET), and detailed neuropsychological evaluation. Moreover, two patients underwent intracranial EEG recordings.

A specific epilepsy protocol, including thin sliced axial and coronal plane T2 weighted, T1 weighted, and fluid attenuated inversion recovery sequences along with a susceptibility weighted imaging sequence, was applied during MRI acquisition. The 3T MRI scanner is being used in our facility since 2010. Seizure classifications were based on thorough clinical assessments, and a strong clinico-anatomo-electrical correlation was sine qua non for surgical indication. Four vessel digital cerebral angiography and ultra-high-resolution C-arm flat detector CT angiography were performed for 39 patients to investigate whether there was an association with developmental venous anomalies (DVAs) at our center since 2010 (14).

The inclusion criteria were as follows: i) patients who underwent surgical removal of CCMs that were responsible for seizures; ii) patients who had been followed-up for at least 2 years after surgery; iii) patients who were operated on by the same institutional surgical team. Patients with CCM without seizures were not included in this study. Furthermore, we did not include patients with brainstem CCMs or patients with CCMs operated on by other surgeons at our institution. Patients were divided into two groups depending on their response to antiepileptic drugs (AEDs) before surgery: i) drugresistant (DRE) group and ii) drug-responsive (DRP) group. The criteria for classifying patients into the DRE group were as following; i) having used at least two different AED regimens, ii) having at least four seizures per month, iii) having seizures for at least 12 months.

Age at seizure onset, age at surgery, sex, duration of epilepsy, seizure frequency before surgery, brain MRI findings, prescribed AEDs, preoperative and postoperative neurological state, postoperative seizure outcomes, and surgical data were documented and analyzed (10).

Patients or their legal caregivers, in cases of children or patients with intellectual disability, gave their informed signed consent and permitted their information to be used for scientific purposes. Associated developmental anomalies, CCM location, and proximity to eloquent cortices were documented based on to imaging studies.

Neuronavigation (StealthStation ${ }^{\mathrm{TM}} \mathrm{S} 7$ System, Medtronic, USA) was used when necessary to precisely locate the lesion. All patients were operated on using microsurgical techniques. The operative strategy was based on clinical semiology, neuropsychological evaluation, anatomical location of the CCMs, and EEG findings. Although the aim was to remove the CCMs along with the surrounding hemosiderin rims, surgical resection was limited at the functional border delineated by intraoperative neuromonitorization (IOM) in some cases. One of the three aforementioned surgical methods was performed in each case.

The postoperative evaluations were based on MRI, EEG and clinical findings in the early postoperative period. Regular postoperative clinical follow-up was scheduled for the third, sixth, and twelfth months; thereafter, annual follow-up was planned for each patient. Seizure outcome was assessed using Engel's classification, and statistical analyses were performed to identify factors related to seizure outcomes (8). Seizures that occurred within the first 24 hours were not included in this analysis if they were not habitual seizures, as we regarded these seizures relevant to the surgery itself. AEDs were started and withdrawn under the supervision of the epileptologist without a standardized algorithm. The protocol for AED tapering was individualized for each patient, and AEDs were generally withdrawn if the patient was seizure free for at least 6 months and if scalp EEG recordings were free of epileptiform potentials.

\section{Statistical Analysis}

The t-test was used for numerical variables after the variances had been found to be equal based on Levene's test, while the chi-square test was used for categorical variables in order to compare the demographical data of the two groups. EEG results, pre-operative seizure type, lateralization, localization, and Engel classes were also assessed for both groups using the chi-square test to determine the presence of statistical significance. The chi-square test was also used to asses any statistically significant difference for Engel groups according to localization. With a similar purpose of finding out a statistically significant difference within different Engel classes, a nonparametric test, the Kruskal-Wallis test, was used for duration of epilepsy, and a parametric method, analysis of variance, was used for localization. $\mathrm{p}<0.05$ was considered statistically significant. All statistical analyses were performed using SPSS (version 22.0; IBM Corp, Armonk, New York, USA).

\section{RESULTS}

We identified 56 patients meeting the eligibility criteria. Male/ female ratio was $26 / 30$, and the mean age at seizure onset 
was 28.88 years (range, $6-54$ years). Six of the patients were under the age of 18 . The patients were divided into DRE $(n=16)$ and DRP $(n=40)$ groups. In DRE group, mean age at seizure onset was 25.9 years (range, 6-41 years); the mean duration of epilepsy was 36.1 months (range, 13-82 months), and the mean age at the time of the operation was 28.3 years (range, 8-45 years). In DRP group, the mean age at seizure onset was 30.5 years (range, 11-54 years), the mean duration of epilepsy was 17.5 months (range, 1-57 months), and the mean age at the time of the operation was 32 years (range, 11-54 years) (Table I).

When the demographical variables between the two groups were assessed, only the duration of epilepsy was found to be significantly different (longer in DRE group, $p<0.001$ ), as expected. There were no other significant differences in terms of any other demographical variables (Table I).

In addition to epileptic seizures, 10 of the patients (18\%) had pre-operative neurological deficits (hemiparesis, sensory deficits, dysphasia, or visual disturbance) that were associated with the location of the CCMs (Table I). There were no additional post-operative neurological deficits, and pre-operative deficits did not change during the early followup period. Long-term improvement was noted in two of the patients who had pre-operative hemiparesis.

DVAs were found in the neighborhood of the CCM in 17 out of 39 patients who underwent four vessel digital cerebral angiography and ultra-high-resolution C-arm flat detector CT angiography.

CCMs were mostly observed in the frontal lobe $(48 \%, 14$ right/13 left), followed by the temporal lobe (32\%, 9 right/9 left), parietal lobe (11\%, 3 right/3 left), occipital lobe (5\%, 2 right/1 left), and insula (4\%, 1 right/1 left). CCM lesions were totally removed in all cases, and the perilesional hemosiderin ring was also totally excised in non-eloquent regions using the micro neurosurgical technique. On the other hand, for lesions located in or near the eloquent cortices, the excision was limited to functional margins using IOM. In five cases, the lesions were removed without extensive resection of the perilesional hemosiderin due to intricate involvement with the eloquent cortex. In 44 patients, the surrounding hemosiderin was removed with the CCMs. Extended lesionectomy was performed in seven cases; in four of these, the lesions were located in the mesial temporal structures. Removal of the perilesional hemosiderin was controlled and confirmed in all patients using early postoperative MRI.

The average follow-up period after resection was 69.6 months (range, 24-216 months). The seizure outcome was assessed according to Engel's classification at the last follow-up. Engel class I was achieved in 53 patients (95\%); there was one patient at class II and two patients at class III. All patients in the DRP group were at Engel class I after the surgery, while all patients at Engel classes II and III were in the DRE patient group. This clearly shows that there were better outcomes in DRP group $(p<0.01)$. Neither the locations of cavernomas nor the duration of epilepsy had any impact on seizure outcome $(p>0.05)$.
The three patients with unfavorable outcome were all adults. The first patient at Engel class III was a 33-year-old woman who presented with daily seizures for the last 23 months due to a left insular CCM. Pure lesionectomy was performed since the surrounding hemosiderin rim could not be removed totally because of the close relation with the corticospinal tract. The number of prescribed AEDs was two in the pre-operative period as well as at post-operative follow-up. Her seizure frequency was 7 to 8 per month at the last follow-up. The second patient at class III was a 30-year-old woman who presented with 2-3 seizures per week. The seizures had started 7 years ago due to a right frontal CCM. This patient had the longest duration of epilepsy among all patients. The hemosiderin rim along with the CCM were removed during surgery, and the number of prescribed AEDs was diminished from two to one. She has had nocturnal seizures once a week since the surgery. The patient with a class II outcome was a 25-year-old man with an average of four seizures per month during last the 13 months due to a left frontal CCM. The number of AEDs was two before surgery, but decreased to one after removal of the CCM. The hemosiderin rim involved the eloquent cortex that limited the resection.

Due to the small number of patients with CCMs in the occipital, insular, and parietal lobes, statistical analysis could be performed only between CCMs in the frontal and temporal lobes and the other locations. No statistically significant difference was found between these groups in terms of seizure outcome $(p>0.05)$.

AEDs were discontinued after surgery in 42 out of 53 seizurefree patients, of whom 11 were in the DRE group. The number of drugs continued as before in two and one patients in DRE and DRP groups, respectively. The treatment of six patients was switched to monotherapy in DRP group. Two patients declined drug withdrawal and continued with monotherapy (Table I).

\section{DISCUSSION}

CCMs are one of the causes of lesion-related epilepsy and are amenable to epilepsy surgery. Our study involved 56 patients with CRE who underwent surgery. Although the current literature is more focused on CCMs in the temporal lobe, it is known that extratemporal locations accounts for $60-70 \%$ of these malformations (18,21). Previous studies reported favorable outcomes in $79-87 \%$ of patients with CCM, whereas the figure increases up to $83-90 \%$ when the CCM is located in the temporal lobe $(12,30-32)$. In our study, CCMs were mostly observed in the frontal lobe (27/56), followed by the temporal lobe (18/56). Considering the involvement of various anatomical locations, we observed a high rate of seizure control in CRE compared to those in previous reports.

Our study supports the efficacy of surgical treatment for CRE. There have been studies with large patient series which argued that it was possible to achieve long-term clinical stability, quality of life, and functional neurological outcome only with AED treatment $(2,16)$. However, our study demonstrated a favorable outcome in $95 \%$ of patients after surgery. Along with 
Table I: Demographic, Clinical, and Anatomical Data and Seizure Outcomes in the DRE and DRP Patient Groups

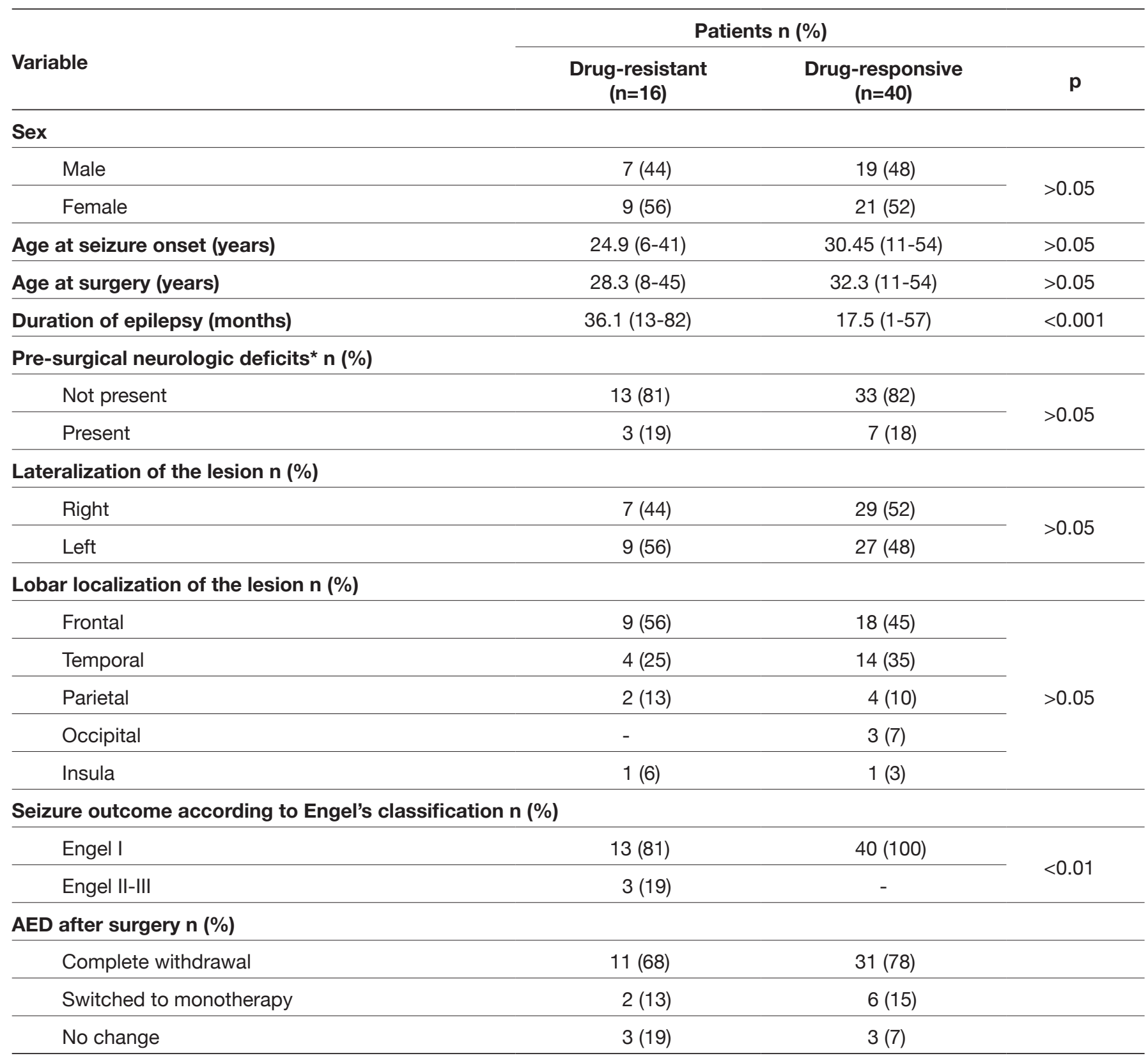

AED: Antiepileptic drug, DRE: Drug resistant, DRP: Drug responsive. *hemiparesis, sensory deficits, aphasia, visual disturbances.

the surgical success for seizure freedom, AED discontinuation or switching to monotherapy may also avoid the impacts of lifelong AED use. In our study, 43 patients discontinued AED therapy, and six patients had tapered down to single AED use at the follow-up.

Seizure outcome can worsen in the long-term compared to that in the early postoperative period (12). However, in our study, all the provided outcome data were according to the last available follow-up (at least 2 years). Although the current study presents long-term seizure outcomes with a mean follow-up of 69.6 months, the favorable outcome (95\% Engel class I) rate was still high.

A considerable number of studies have indicated that the microhemorrhages into the surrounding brain parenchyma that are caused by CCMs are strongly associated with the epileptic seizures. It is thought that, following perilesional hemosiderosis, reactive gliosis and the cortical scarring due to the CCM contribute to the chronic seizures. We believe that the removal of the hemosiderin rim around the CCM is necessary in modern CCM surgery. Strong clinical evidence has been 
provided in a review article by Dammann et al. supporting this hypothesis regarding perilesional hemosiderin containing tissue (7). Furthermore, the fact that a longer preoperative duration of epilepsy was associated with worse postoperative seizure outcomes (12) could be attributed to extending of the epileptogenic zone network with time. Increasing the preoperative duration might result in more drug-resistant epilepsy cases, more AED use, and less seizure freedom after future possible surgeries. Although our results did not show a direct relation between a shorter duration of epilepsy and better outcomes in CRE, we did find a correlation between drug resistance and longer duration of epilepsy. Therefore, it seems that early surgical removal of CCMs could be a factor resulting in favorable seizure outcomes.

For the lesions located in the eloquent regions, utilization of modern neurosurgical modalities like neuronavigation, preoperative functional $\mathrm{MRI}$, diffusion tensor imaging, and IOM seems essential to overcome the challenge of balancing extensive resection and possible neurological sequelae. None of the five patients who had CCMs in the eloquent regions and were treated using these modalities in the current study had an additional long-term neurological deficit.

The three patients whose seizures continued in the postoperative period were in the DRE group, and the lesions were in close proximity to the eloquent cortices. Minimal residual hemosiderin containing tissue was left at the functional boundaries in two patients. As is consistent with the literature, resection involving the hemosiderin rim is an important prognostic factor for seizure outcome, which might be complicated in CCMs at or near eloquent cortices due to residual hemosiderin. A long history and high seizure frequency were remarkable in the other patient. The extensive epileptogenic zone network might explain the persistence of seizures despite complete resection of the lesion and the hemosiderin rim.

For CCMs that involve the temporal lobe, extended lesionectomy may be required to ensure that the seizures cease (24). Chronic exposure to seizure activity may also contribute to secondary epileptogenesis $(3,27)$. Some studies suggest removal of epileptogenic mesial temporal structures $(27,28)$. In spite of these factors and studies providing a clear mechanism and data in favor of extended resection, ShangGuan et al.'s meta-analysis concluded that there is no evidence to prove that extended lesionectomy for CCMs results in better seizure control than lesionectomy (25). As the excised region of brain parenchyma is increased in extended lesionectomy, a more detailed assessment should be carried out to weigh the possible risks associated with postoperative neurocognitive deficits and the potential benefits against each other. Furthermore, some studies suggest a two-step approach for treating temporal CCMs to avoid unnecessary hippocampal removal-the first step involves pure lesionectomy, while a possible second step amygdalohippocampectomy may be performed if there is no improvement in seizure outcome $(1,9)$. However, we believe that this two-step approach would be unnecessary for most patients with hippocampal formations involved in the epileptogenic network. For CREs that involve the temporal lobe, our surgical strategy depends on the exact location of the lesion, memory impairment, whether seizure history was recent, and whether seizures were few or chronic. Patients without chronic seizures underwent lesionectomy regardless of the location when memory was intact. However, mesial structures were removed if the memory was affected. In chronic patients with CRE and CCMs located in the lateral temporal lobe, mesial structures were spared if the memory was intact; otherwise, they were removed. Mesial structures were removed in chronic seizure patients with mesially located CCMs regardless of memory status.

The retrospective design and limited number of patients are the principal limitations of the present study. Several questions related to surgical technique can only be answered by performing prospective studies with larger patient cohorts in the future.

In summary, we present a retrospective CRE study which covers all cerebral lobes. The approach to CREs should be tailored to each patient according to the clinical findings and presurgical work-up for delineation of the epileptogenic network. Surgical intervention for CCMs may have favorable outcomes regardless of the localization of the lesion and the duration of epilepsy. However, an earlier surgical intervention may prevent the patients from becoming drug-resistant such that their chances of being seizure free after surgery increase.

\section{REFERENCES}

1. Awad I, Jabbour P: Cerebral cavernous malformations and epilepsy. Neurosurg Focus Jul 21(1):e7, 2006

2. Bicalho VC, Bergmann A, Domingues F, Frossard JT, De Souza JPBM: Cerebral cavernous malformations: Patient-reported outcome validates conservative management. Cerebrovasc Dis 44:313-319, 2017

3. Blümcke I, Thom M, Aronica E, Armstrong DD, Vinters HV, Palmini A, Jacques TS, Avanzini G, Barkovich AJ, Battaglia G, Becker A, Cepeda C, Cendes F, Colombo N, Crino P, Cross JH, Delalande O, Dubeau F, Duncan J, Guerrini R, Kahane P, Mathern G, Najm I, Ozkara C, Raybaud C, Represa A, Roper SN, Salamon N, Schulze-Bonhage A, Tassi L, Vezzani A, Spreafico R: The clinicopathologic spectrum of focal cortical dysplasias: A consensus classification proposed by an ad hoc Task Force of the ILAE Diagnostic Methods Commission. Epilepsia 52:158-174, 2011

4. Campbell PG, Jabbour P, Yadla S, Awad IA: Emerging clinical imaging techniques for cerebral cavernous malformations: A systematic review. Neurosurg Focus 29(3):E6, 2010

5. Chalouhi N, Dumont AS, Randazzo C, Tjoumakaris S, Gonzalez LF, Rosenwasser R, Jabbour P: Management of incidentally discovered intracranial vascular abnormalities. Neurosurg Focus 31(6):E1, 2011.

6. Chang EF, Gabriel RA, Potts MB, Garcia PA, Barbaro NM, Lawton MT: Seizure characteristics and control after microsurgical resection of supratentorial cerebral cavernous malformations. Neurosurgery 65(1):31-37; discussion 37-38, 2009 
7. Dammann P, Schaller C, Sure U: Should we resect perilesional hemosiderin deposits when performing lesionectomy in patients with cavernoma-related epilepsy (CRE)? Neurosurg Rev 40:39-43, 2017

8. Engel J: Update on surgical treatment of the epilepsies. Clin Exp Neurol 29:32-48, 1992

9. Ferroli P, Casazza M, Marras C, Mendola C, Franzini A, Broggi G: Cerebral cavernomas and seizures: A retrospective study on 163 patients who underwent pure lesionectomy. Neurol Sci 26:390-394, 2006

10. Fisher RS, Cross JH, French JA, Higurashi N, Hirsch E, Jansen FE, Lagae L, Moshé SL, Peltola J, Roulet Perez E, Scheffer IE, Zuberi SM: Operational classification of seizure types by the International League Against Epilepsy: Position paper of the ILAE commission for classification and terminology. Epilepsia 58(4):522-530, 2017.

11. Hauck EF, Barnett SL, White JA, Samson D: Symptomatic brainstem cavernomas. Neurosurgery 64(1):61-70; discussion 70-71, 2009

12. He K, Jiang S, Song J, Wu Z, Chen L, Mao Y: Longterm outcomes of surgical treatment in 181 patients with supratentorial cerebral cavernous malformation-associated epilepsy. World Neurosurg 108:869-875, 2017

13. Kashida Y, Usui N, Matsuda K, Terada K, Baba K, Kondo A, Hirozawa D, Tottori T, Mihara T, Hanaya R, Arita K, Inoue Y: Is additional mesial temporal resection necessary for intractable epilepsy with cavernous malformations in the temporal neocortex? Epilepsy Behav 92:145-153, 2019

14. Kocak B, Kizilkilic O, Oz B, Bakkaloglu DV, Isler C, Kocer $\mathrm{N}$, Islak C: Ultra-high-resolution C-arm flat-detector CT angiography evaluation reveals 3-fold higher association rate for sporadic intracranial cavernous malformations and developmental venous anomalies: A retrospective study in consecutive 58 patients with 60 cavernous malformations. Eur Radiol 27:2629-2639, 2017

15. Kondziolka D, Monaco EA, Lunsford LD: Cavernous malformations and hemorrhage risk. Prog Neurol Surg 27:141146, 2013

16. Lee Y, Cho KH, Kim HI, Lee SK, Cho YJ, Heo K, Lee BI: Clinical outcome following medical treatment of cavernous malformation related epilepsy. Seizure 45:64-69, 2017

17. Maraire JN, Awad IA: Intracranial cavernous malformations: Lesion behavior and management strategies. Neurosurgery 37(4):591-605, 1995

18. Moran NF, Fish DR, Kitchen N, Shorvon S, Kendall BE, Stevens JM: Supratentorial cavernous haemangiomas and epilepsy: A review of the literature and case series. J Neurol Neurosurg Psychiatry 66:561-568, 1999

19. Moriarity JL, Clatterbuck RE, Rigamonti D: The natural history of cavernous malformations. Neurosurg Clin N Am 10:411417, 1999

20. Robinson JR, Awad IA, Little JR: Natural history of the cavernous angioma. Journal Neurosurg 75(5):709-714, 1991

21. Rocamora R, Mader I, Zentner J, Schulze-Bonhage A: Epilepsy surgery in patients with multiple cerebral cavernous malformations. Seizure 18:241-245, 2009
22. Rosenow F, Alonso-Vanegas MA, Baumgartner C, Blümcke I, Carreño M, Gizewski ER, Hamer HM, Knake S, Kahane P, Lüders HO, Mathern GW, Menzler K, Miller J, Otsuki T, Ozkara C, Pitkänen A, Roper SN, Sakamoto AC, Sure U, Walker MC, Steinhoff BJ; Surgical Task Force, Commission on Therapeutic Strategies of the ILAE: In response to commentary on cavernoma-related epilepsy: Review and recommendations for management-report of the surgical task force of the ILAE commission on therapeutic strategies. Epilepsia 55(3):466467, 2014

23. Salman R, Hall JM, Horne MA, Moultrie F, Josephson CB, Bhattacharya JJ, Counsell CE, Murray GD, Papanastassiou V, Ritchie V, Roberts RC, Sellar RJ, Warlow CP; Scottish Audit of Intracranial Vascular Malformations (SAIVMs) collaborators: Untreated clinical course of cerebral cavernous malformations: A prospective, population-based cohort study. Lancet Neurol 11(3):217-224, 2012

24. Schuss P, Marx J, Borger V, Brandecker S, Güresir Á, Hadjiathanasiou A, Hamed M, Schneider M, Surges R, Vatter $H$, Güresir E: Cavernoma-related epilepsy in cavernous malformations located within the temporal lobe: Surgical management and seizure outcome. Neurosurg Focus Apr 48(4):E6, 2020

25. Shang-Guan HC, Wu ZY, Yao PS, Chen GR, Zheng SF, Kang DZ: Is extended lesionectomy needed for patients with cerebral cavernous malformations presenting with epilepsy? A meta-analysis. World Neurosurg 120:e984-e990, 2018

26. So EL, Radhakrishnan K, Silbert PL, Cascino GD, Sharbrough FW, O'Brien PC: Assessing changes over time in temporal lobectomy: Outcome by scoring seizure frequency. Epilepsy Res 27(2):119-125, 1997

27. Upchurch K, Stern JM, Salamon N, Dewar S, Engel J Jr, Vinters HV, Fried I: Epileptogenic temporal cavernous malformations: Operative strategies and postoperative seizure outcomes. Seizure 19:120-128, 2010

28. Vale FL, Vivas AC, Manwaring J, Schoenberg MR, Benbadis SR: Temporal lobe epilepsy and cavernous malformations: Surgical strategies and long-term outcomes. Acta Neurochir (Wien) 157:1887-1895, 2015

29. Wang CC, Liu A, Zhang JT, Sun B, Zhao YL: Surgical management of brain-stem cavernous malformations: Report of 137 cases. Surg Neurol 59(6):444-454, 2003

30. Yang PF, Pei JS, Jia YZ, Lin Q, Xiao H, Zhang TT, Zhong ZH: Surgical management and long-term seizure outcome after surgery for temporal lobe epilepsy associated with cerebral cavernous malformations. World Neurosurg 110:e659-e670, 2018

31. Yeon JY, Kim JS, Choi SJ, Seo DW, Hong SB, Hong SC: Supratentorial cavernous angiomas presenting with seizures: Surgical outcomes in 60 consecutive patients. Seizure 18(1):14-20, 2009

32. Zevgaridis D, Van Velthoven V, Ebeling U, Reulen HJ: Seizure control following surgery in supratentorial cavernous malformations: A retrospective study in 77 patients. Acta Neurochir (Wien) 138(6):672-677, 1996 